\title{
LINC00466 Impacts Cell Proliferation, Metastasis and Sensitivity to Temozolomide of Glioma by Sponging miR-I 37 to Regulate PPPIR I4B Expression
}

This article was published in the following Dove Press journal: OncoTargets and Therapy

\author{
Mingfei Zhao',* \\ Yijie Shaol,* \\ Jinfang Xu (D) \\ Buyi Zhang ${ }^{2}$ \\ Chenguang $\mathrm{Li}^{1}$ \\ Jie Gong ${ }^{3}$
}

'Department of Neurosurgery, Second Affiliated Hospital, School of Medicine, Zhejiang University, Hangzhou, Zhejiang, People's Republic of China; ${ }^{2}$ Department of Pathology, Second Affiliated Hospital, School of Medicine, Zhejiang University, Hangzhou, Zhejiang, People's Republic of China; ${ }^{3}$ Department of Neurointerventional, Zhejiang Hospital of Zhejiang Province, Hangzhou, Zhejiang, People's Republic of China

*These authors contributed equally to this work
Purpose: LINC00466 is a newfound long non-coding RNA (lncRNA) that has been rarely explored in cancers. However, the specific role and molecular mechanism of LINC00466 in glioma remain to be further elucidated.

Methods: Bioinformatic analysis was used to screen differentially expressed genes. Quantitative real-time PCR (qRT-PCR) was used to determine the expression of LINC00466, microRNA-137 (miR-137) and protein phosphatase 1 regulatory subunit 14B (PPP1R14B). Dual-luciferase reporter gene assay and RNA binding protein Immunoprecipitation (RIP) assays were employed to verify the binding relationship among LINC00466, miR-137 and PPP1R14B. The sensitivity of glioma cells to temozolomide (TMZ) was measured by cell counting kit- 8 (CCK8) assay. The xenograft nude models were used to test the effects of LINC00466 on glioma tumor growth in vivo.

Results: Highly expressed LINC00466 and PPP1R14B and lowly expressed miR-137 were eventually revealed in glioma tissues. Overexpression of LINC00466 could promote proliferation, metastasis and drug sensitivity to TMZ of glioma cells. LINC00466 could bind to miR-137, and up-regulation of miR-137 could attenuate the enhancing effects caused by LINC00466 overexpression. We took a further step and found that miR-137 could bind to PPP1R14B. Besides, LINC00466 could function as a sponge to miR-137 to regulate PPP1R14B. In addition, overexpression of LINC00466 could promote tumor growth in vivo. Conclusion: These findings validate LINC00466 could restrain the miR-137 expression to up-regulate PPP1R14B and therefore promote proliferation, metastasis and resistance to TMZ of glioma.

Keywords: LINC00466/miR-137/PPP1R14B, glioma, proliferation and metastasis, temozolomide; TMZ

\section{Introduction}

Glioma is one of malignant tumors which can cause an adverse effect on the brain and spine. ${ }^{1,2}$ Mixed tumors, ependymoma, oligodendroglioma and astrocytoma are four common subtypes of this kind of tumor. ${ }^{3}$ The treatment methods of glioma focus on surgery, radiotherapy, and chemotherapy. These methods can improve the physical condition of patients. However, the infiltrative growth characteristics and chemotherapy resistance of glioma have brought challenges to the treatment of glioma. ${ }^{4}$ In response to these challenges, scientists have conducted in-depth research into the molecular mechanisms underlying glioma development and progression. Even so, the uncovered molecular mechanisms underlying glioma
Correspondence: Jie Gong

Tel +86-I5I68248628

$\mathrm{Fax}+8657 \mathrm{I}-88098 \mathrm{I} 23$

Email orchidwave@I63.com 
development and progression were limited so far. ${ }^{5}$ Therefore, an in-depth understanding of the underlying molecular and biological mechanisms of glioma progression will help us to develop new therapeutic and prognostic approaches.

Non-coding RNAs contribute to the pathogenesis of different cancers, including glioma. Non-coding RNAs are almost involved in the proliferation, differentiation and metastasis of tumor cells. In addition, non-coding RNAs are constantly being discovered as biomarkers, and these discovered non-coding RNAs may serve as targets for tumor therapy. ${ }^{6}$ In the study of the occurrence and prognosis of glioma, the dysregulation of long noncoding RNAs (lncRNAs) has been recently considered to be associated with the occurrence and development of glioma. ${ }^{7,8}$ As for the correlation between lncRNAs and cancer, numerous studies have confirmed that lncRNAs play a crucial role in complex biological processes of glioma cells. LncRNAs can participate in gene regulation through various mechanisms, including competing endogenous RNA (ceRNA) mechanisms. Besides, IncRNAs possess miRNA Responsible Elements (MRE), the binding sites with miRNAs by which they can potentially sponge miRNAs that lead to miRNAmediated post-transcriptional regulation of target mRNAs. ${ }^{9,10}$ Meng et al found that lncRNA SNHG5 acted as a ceRNA in glioma to regulate ZEB2 by competitively binding to $\mathrm{miR}-205-5 \mathrm{p}$, thereby affecting biological function of glioma. ${ }^{11}$ Liu et al proved that lncRNA H19 could promote glioma angiogenesis through miR-138/HIF-1 $\alpha /$ VEGF axis. ${ }^{12}$ The discovery of these lncRNA-miRNA-mRNA signaling axes helps to the prognosis and diagnosis of glioma. However, studies concerning the lncRNA-miRNA-mRNA signaling axis remain scarce.

Temozolomide (TMZ), an alkylating agent of the imidazoline series, is a first-line chemotherapy drug in clinical treatment of malignant gliomas. After oral administration, it is absorbed rapidly and enters the cerebrospinal fluid through the blood-brain barrier, reaching an effective blood drug concentration in the central nervous system. ${ }^{13}$ However, the resistance of $\mathrm{TMZ}$ to glioma poses heavy burden to glioma treatment. ${ }^{14}$ Some researchers found that certain lncRNAs such as SNHG15, NCK1-AS1 and MALAT1 could affect the sensitivity of glioma cells to TMZ chemotherapy. ${ }^{13,15,16}$ However, the roles of other lncRNAs in pathogenesis and TMA resistance of glioma are still unclear.
In this study, we first found that LINC00466 could promote proliferation, migration and invasion of glioma cells. Using bioinformatic analyses, we found that LINC00466 may affects glioma cells through miR-137/ PPP1R14B axis. Based on this, we aimed to investigate the expression, functional role, TMZ resistance and underlying mechanism of LINC00466 in glioma. Our results suggest LINC00466 can affect cell viability and TMZ resistance of glioma cells by regulating miR-137/ PPP1R14B axis.

\section{Materials and Methods Human Tissue Processing}

The fresh original glioma tissues, adjacent non-cancerous were acquired (number $=30$, including 11 males and 19 females) after surgery. The patients which have treated by surgery between December 2018 and June 2020 in the second affiliated hospital of Zhejiang university and signed written informed consent. The ethics committee of the second affiliated hospital of Zhejiang university have consented the research (Approval No. 2020549, Supplement Figure1). Supplement Table 1 showed the clinicopathological data of the patients.

\section{Cell Lines}

All commercial cells used in this study were detailed in Supplement Table 2. Dulbecco's modified Eagle medium (DMEM; Gibco, Grand island, NY, USA) supplemented with $10 \%$ fetal bovine serum (Gibco), $100 \mathrm{U} / \mathrm{mL}$ penicillin and $100 \mathrm{mg} / \mathrm{mL}$ streptomycin (Gibco) were used to culture cell. Cells were cultured at $37^{\circ} \mathrm{C}$ in a humidified atmosphere of $5 \% \mathrm{CO}_{2}$.

\section{Cell Transfection}

Cloned full-length sequence of LINC00466, LINC00466 overexpression plasmid and pcDNA3.1 vector were accessed from Genechem Co., LTD. After achieving $70 \%$ confluence in a well, treated cells were used for subsequent analysis. After the lentiviral transduction, cell lines were treated with $1 \mathrm{mg} / \mathrm{mL}$ purinomycin to obtain stably transfected cell lines. miR-137 mimic (miR-137), sh-miR-137, si-PPP1R14B and their corresponding controls (NC) were purchased form Ribo Co., Ltd. (Guangzhou, china). Lipofectamine 3000 (L3000015, Invitrogen, USA) was used to transfected cells following the standard process. 


\section{Real-Time Quantitative PCR (qRT-PCR)}

TRIzol reagent (Takara bio, Otsu, Japan) was used to extract total RNA from cells or human tissue following the standard process. All-in-One First-Strand cDNA Synthesis kit (GeneCopoeia, Rockville, MD, USA) was used to synthesized cDNA. qRT-PCR was performed on ABI 7900HT instrument (Applied Biosystems, USA). LncRNA and mRNA levels were detected by using the Sybr Green PCR Master Mix and miRNA levels were detected by using Miscript SYBR Green PCR Kit (Qiagen, Germany). LINC00466, PPP1R14B and miR137 were normalized to GAPDH and U6, respectively. Supplement Table 3 listed the primers which were used in this study. $2^{-\Delta \Delta \mathrm{Ct}}$ method was used to analyze the results. The research was performed three times.

\section{Western Blot (WB) Analysis}

The samples of tissues and cells were lysed with Radio Immune Precipitation Assay (RIPA) lysis buffer (Beyotime Institute of Biotechnology, Haimen, china). SDS-PAGE was used to separated proteins and proteins were transferred to Polyvinylidene Fluoride (PVDF) membranes (Roche, Basel, Switzerland). Five percent Bovine Serum Albumin or tris-buffered saline tween (BSA/TBST) were used to block the PVDF membranes. Then, primary antibodies were used to incubate the membranes overnight at $4{ }^{\circ} \mathrm{C}$. Next, $1 \times$ TBST (Solarbio, Beijing, china) was employed to wash the membranes at room temperature. Horseradish Peroxidase (HRP)-labeled secondary antibody goat anti-rabbit IgG was sequentially added into the membranes for incubation for $115 \mathrm{~min}$ at temperature of room. Membranes were washed with TBST three times, $20 \mathrm{~min}$ each time. Electrochemiluminescence (ECL) kit (Solarbio, Beijing, China) was used to visualized proteins bands. The experiment was made in three times. Supplement Table 4 lists the information of antibodies.

\section{RNA Binding Protein Immunoprecipitation}

Magna RNA immunoprecipitation (RIP) kit (Millipore, USA) was used to complete RIP assay in T98G cells. The cell lysate was cultured in RIP buffer containing magnetic beads and then combined with human rabbit anti-Ago2 antibody. Input was used as positive control. IgG was used as negative control. Protease $\mathrm{K}$ was used to purify and immune-precipitate RNA. The sample and Input were digested by protease $\mathrm{K}$ and then RNAs
(LINC00466 and PPP1R14B) were extracted for subsequent qRT-PCR analysis. All the antibodies used were listed in Supplement Table 4.

\section{Cell Viability Assay and Colony Formation Assay}

Cell Counting Kit-8 (CCK-8) (Dojindo, Kumamoto, Japan) was used to analyze cell proliferation. Cells were cultured in 96-well plates for $1 \mathrm{~d}, 2 \mathrm{~d}$ and $3 \mathrm{~d}$, respectively. $100 \mu \mathrm{L}$ T98G or A172 cell lines were planted into 96-well plates at a density of $5 \times 10^{3}$ cells $/ 100 \mu \mathrm{L}$. Each treatment was conducted in triplicate. The absorbance value at 450 $\mathrm{nm}$ was measured by an enzyme-labeled instrument (Molecular Devices, Sunnyvale, CA, USA). For TMZ experiments, cells were seeded in 96-well plates and cultured for $1 \mathrm{~d}$, followed by treatment with $200 \mathrm{uM} \mathrm{TMZ}$ for another $2 \mathrm{~d}$. For colony formation assay, $1 \times 10^{3}$ cells/well T98G or A172 cells were added into plates with 6 repeated wells for each treatment. After 14 days, the culture medium was replaced. The colonies were fixed in $30 \%$ Formaldehyde (thermo fisher, USA) for $15 \mathrm{~min}$, and then stained with $0.1 \%$ Crystal Violet (thermo fisher, USA).

\section{Transwell Assay}

T98G and A172 cells in logarithmic growth stage were starved for $24 \mathrm{~h}$, and the cells were digested, centrifuged and resuspended on next day. The upper Chambers of the Transwell filter chamber are coated with Matrigel or not (50 $\mu \mathrm{L}, 356234$, BD, franklin lakes, NJ) per well. The cells were inoculated into the apical cavity of the Transwell filter and incubated in serum-free medium for 24 hours (medium containing 10\% fetal bovine serum was added to the basolateral cavity). The invasive/transitional cells below the filter were fixed with $5 \%$ glutaraldehyde, stained with $0.1 \%$ crystal violet, and counted microscopically. The invasion/migration ability of each group was determined by the number of cells in the gum.

\section{Dual-Luciferase Assay}

Psicheck Luciferase Reporter vectors (Sangon co., LTD, shanghai, china) ligated with Wild-Type (WT) and Mutant-Type (MUT) PPP1R14B/LINC00466 3'untranslated-regions (3'UTR) were established. Thereafter, HEK293T cells (Thermo Fisher, USA) were added into 48 -well plates for $24 \mathrm{~h}$ of incubation. miR-137/ NC and PPP1R14B/LINC00466 WT-vector/MUT-vector were co-transfected into cells. Dual-luciferase reporter 
assay system (Promega) was used to analyze the results. Experiments were repeated at least three times.

\section{Fluorescence in situ Hybridization (FISH) and Subcellular Fractionation}

FISH assay was performed using ribo ${ }^{\text {TM }}$ fluorescent in situ hybridization Kit (Ribobio company, china). For FISH assay, fluorescent probe for LINC00466 was purchased from Ribo Co., Ltd. (Guangzhou, China). The result was observed under a fluorescence microscope (Thermo Fisher, USA) and the images were taken. Nuclear and cytoplasmic separation in A172 cells was performed by using PARIS Kit (Life Technologies, Carlsbad, USA), and then qRTPCR was implemented to detect the expression of LINC00466 and reference genes (U6 and GAPDH) in nucleus and cytoplasm.

\section{Immunohistochemical (IHC) Analysis}

IHC assay was performed as Ho et al recommended. ${ }^{17}$ In brief, paraffin-embedded tissue sections were deparaffinized in xylene and treated with ethanol in gradient concentration. The endogenous peroxidase was inactivated using PBS with $1 \% \mathrm{H}_{2} \mathrm{O}_{2}$ for $10 \mathrm{~min}$, after which the sections were washed with PBS three times and incubated with $5 \%$ skim milk/PBS for $30 \mathrm{~min}$ to decrease nonspecific binding. The sections were subsequently diluted with 5\% skim milk/PBS and incubated with antiPPP1R14B and Ki-67 antibodies for $16 \mathrm{~h}$ at $4{ }^{\circ} \mathrm{C}$. After being washed with PBS twice, the sections were detected by IHC detection system. 3, 3'-diaminobenzidine liquid substrate (Sigma-Aldrich, USA) was used for observation of specific immunostaining, followed by hematoxylin for counterstaining. Non-immune IgG in the same concentration was used as negative control. The information of all antibodies in the experiment was shown in Supplement Table 4.

\section{Nude Mouse Transplantation Tumor Experiment}

Female nude mice were subcutaneously injected in the underarm area with a suspension of $1 \times 10^{7}$ cells in $200 \mathrm{ul}$ of PBS. The mice were observed 4 weeks before killing and recovery of the tumors. Tumor growth was measured after one week. The formula: Volume $\left(\mathrm{cm}^{3}\right)=$ (length $\times$ width $\left.^{2}\right) / 2$ was used to measured tumor volume. The ethics committee of the second affiliated hospital of zhejiang university have consented the research (Approval No.
2020106, Supplement Figure2). Animal studies were performed in compliance with the ARRIVE guidelines. All animals received humane care according to the National Institutes of Health (USA) guidelines.

\section{Statistical Analysis}

SPSS 22.0 software (SPSS; Lnc, Chicago, IL, USA) was used to analyze all data. Measurement data were expressed in Mean \pm Standard Deviation (SD). The data of two groups were compared by $t$ test, whereas multiple group data were compared with One-Way ANOVA. The relationship between LINC00466 expression and clinicopathological data parameters were analyzed by $\chi^{2}$ test. $P<0.05$ was considered highly significant, $P<0.01$ and $P<0.001$ was considered extremely significant.

\section{Results}

\section{LINC00466 Expression is Increased in Glioma Tissues and Cell Lines}

Previously, increased level of LINC00466 has been linked to malignant characteristics of cancer cells ${ }^{18,19}$ but its role in glioma has not been fully appreciated. Here, we first compared the level of LINC00466 between normal brain tissue and glioma tissue (TCGAGBM/LGG) and found that LINC00466 was significantly higher in the glioma samples as compared to that in the normal brain (Figure 1A and B). The expression level of LINC00466 between GBM and LGG was also compared with the data of TCGA and GTEx databases. The results showed the expression level of LINC00466 has no difference between GBM and LGG (Figure 1C). The results showed that the LINC00466 expression level was higher in glioma tissues compared with paired adjacent tissues (Figure 1D, $\mathrm{P}<0.01$ ). For WHO grade, the expression level of LINC00466 was significantly increased in WHO grade (III-IV) compared with WHO grade (I-II) (Figure 1E, $\mathrm{P}<0.05$ ). Meanwhile, the expression of LINC00466 was also elevated in glioma cell lines (LN308, T98G, A172) compared with normal astroglia cell lines (HEB) (Figure 1F, all $\mathrm{P}<0.001)$. Furthermore, in order to judge the function of LINC00466 we detected the fraction of LINC00466 and showed that LINC00466 located in both cytoplasm and nucleus (Figure $1 \mathrm{G}$ and $\mathrm{H}$ ). Overall, these findings indicated that LINC00466 were high expression in glioma and showed the function of ceRNA. 
A

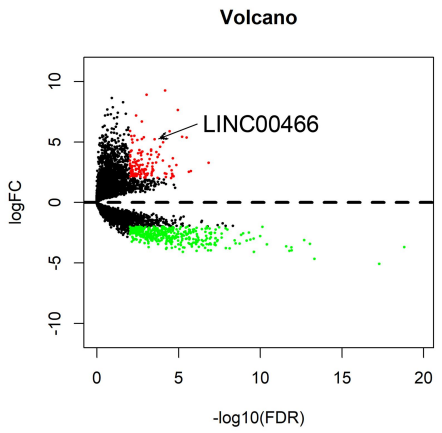

D

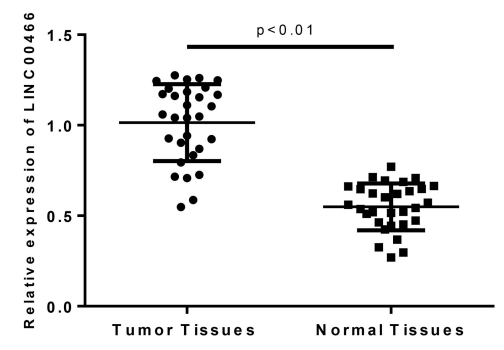

G

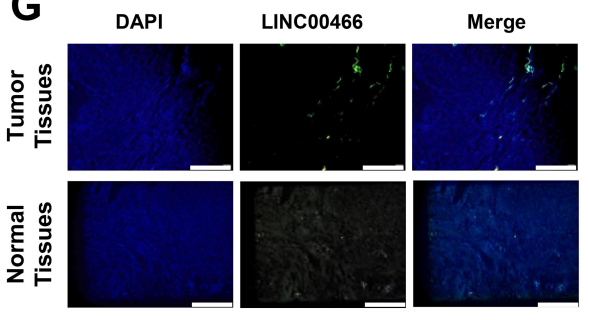

B

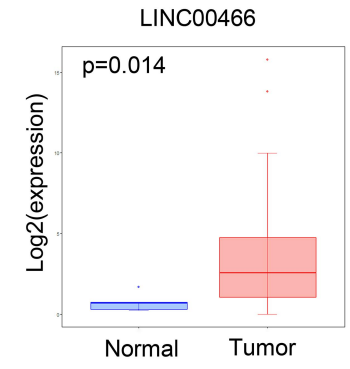

E

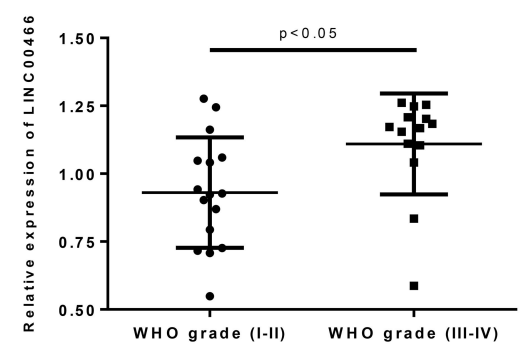

H

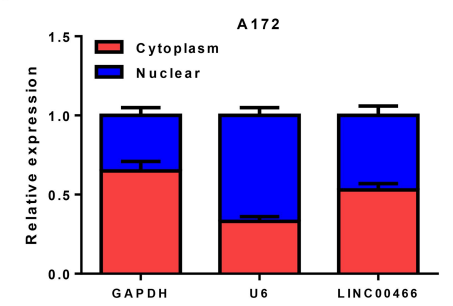

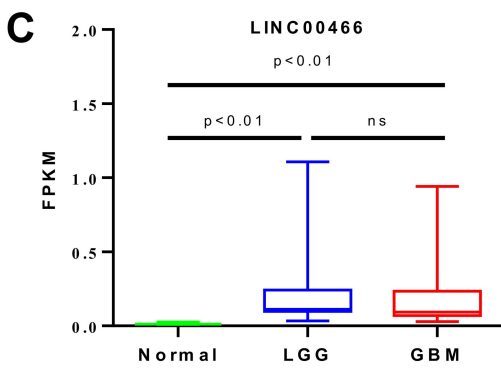

F

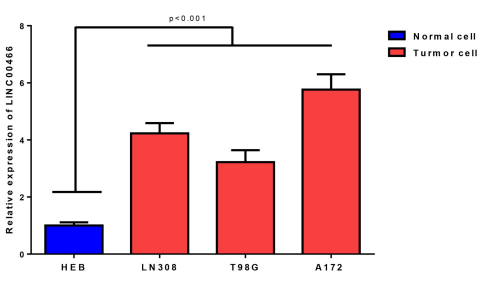

Figure I The expression of LINC00466 is highly in tissues and cell lines of glioma. (A) Volcano plots of the DElncRNAs in TCGA-LGG/GBM database (up-regulated genes were defined as red and down-regulated genes were defined as green); (B) Differential expression of LINC00466 in Tumor and Normal of TCGA-GBM/LGG; (C) Differential expression of LINC00466 among Normal, LGG and GBM of the data in TCGA and GTEx database; (D) The expression level of LINC00466 in the tissue of glioma and adjacent normal tissues was detected by qRT-PCR; (E) The expression of LINC00466 in glioma of WHO grade (I-II) and WHO grade (III-IV); (F) The expression of LINC00466 in three glioma cell lines and one astroglia cell line was detected by qRT-PCR; (G) The location of LINC00466 in glioma tissues was identified by FISH experiments; (H) the expression levels of GAPDH (cytoplasmic marker), U6 (nuclear marker) and LINC00466 after the separation of cell nucleus and cytoplasm in AI72 cells were examined.

\section{LINC00466 Promotes Glioma Cells Activity and Cell Resistance to TMZ}

LINC00466 was found to play an active role in tumorigenesis based on its expression in clinical tissues and cells of glioma. Therefore, we reasoned that up-regulation of LINC00466 could promote the activity of glioma cells. To validate the possibility, we examined the effects of overexpressing LINC00466 on glioma cell proliferation, migration and invasion. T98G and A172 cell lines were used for subsequent cell experiments. Oe-LINC00466 and negative control were transfected into T98G and A172 cells, respectively. Transfection efficiency was detected to be well in cells transfected with oeLINC00466, as suggested by qRT-PCR plotted in Figure 2A. CCK8 assay and colony formation assay showed that upregulation of LINC00466 considerably facilitated the viability of T98G and A172 cells (Figure 2B and C). We further evaluated the effect of LINC00466 on migration and invasion abilities of glioma cells by transwell assays, finding that their abilities were remarkably increased after overexpression of LINC00466 (Figure 2D and E). These findings validated that LINC00466 could act as an oncogene to promote proliferation, migration and invasion of glioma cells. We then investigated the effects of TMZ on T98G and A172 cells. As shown in Figure 2F, using CCK8 assays, treatment with LINC00466 significantly attenuated TMZ cytotoxicity in T98G and A172 cells. These results implied that LINC00466 could promote glioma cell activity and cell resistance to TMZ.

\section{LINC00466 Functions as a Sponge for miR-I 37 in Glioma Cells}

To explore whether miRNAs participate in LINC00466mediated glioma cell proliferation, migration, invasion and 


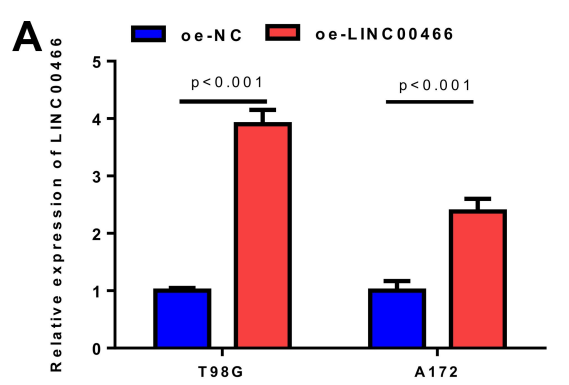

C

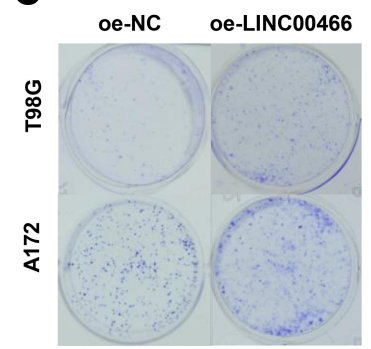

E

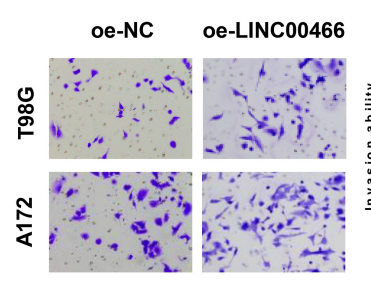

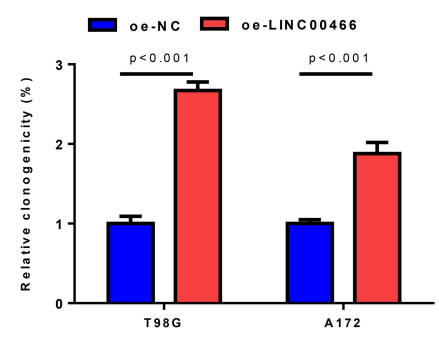

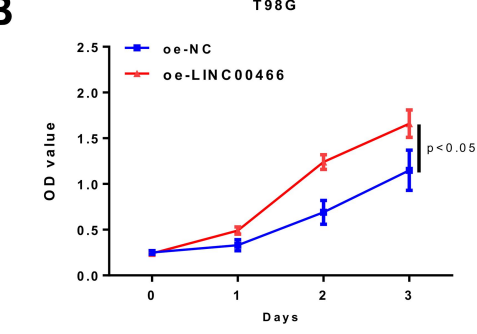

D

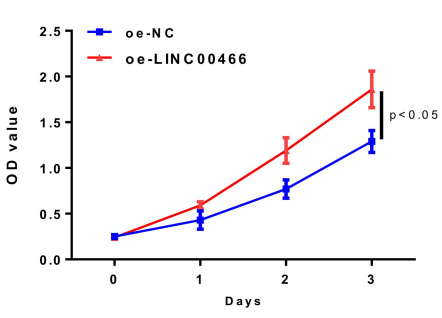

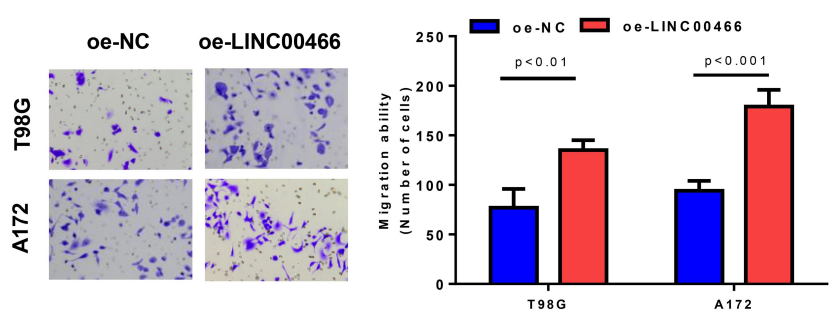

$\mathbf{F}$

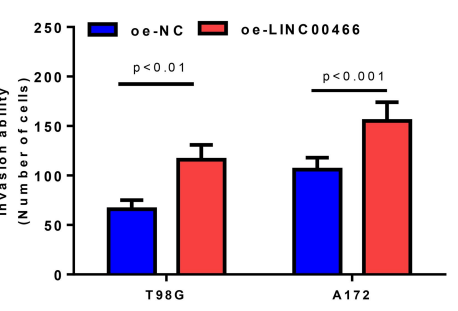

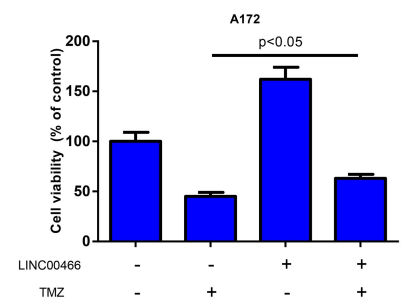

Figure 2 LINC00466 can promote glioma cell activity and resistance to TMZ. (A) The expression of LINC00466 in T98G and AI72 cells treated with oe-NC and oeLINC00466; (B) The viability, (C) colony formation, (D and E) migration combined with invasion of T98G and AI72 cells with overexpression of LINC00466 were detected by CCK8 assay, colony formation assay and Transwell assay; (F) Effects of LINC00466 on the resistance of glioma cells to TMZ (200uM).

sensitivity to TMZ treatment, we conducted a differential analysis using the miRNA expression profile from TCGAGBM/LGG and used miRcode database to predict the miRNAs regulated by LINC00466. The target miRNA of miR-137 was obtained (Figure 3A). miR-137 expression was significantly down-regulated in tumor tissues (Figure 3B and C). Dual-luciferase Reporter gene assay showed that miR-137-mimic inhibited the luciferase activity of LINC00466-WT, while had no influence on LINC00466MUT, suggesting there was a targeted relationship between miR-137 and LINC00466 (Figure 3D). RIP experiments were performed with $\mathrm{T} 98 \mathrm{G}$ and A172 cell lines and further validated their relationship (Figure 3E). In addition, we detected the effects of LINC00466 overexpression on the expression level of miR-137 in T98G and A172 cell lines, showing that miR-137 was significantly decreased in cells upon overexpression of LINC00466 (Figure 3F). Taken together, these findings displayed that LINC00466 could serve as a sponge to bind with miR-137.
In order to prove that LINC00466 could regulate cell biological functions by binding to miR-137, we divided cell lines into 3 treatment groups (oe-NC+miR-NC, oe-NC + miR-mimic, oe-LINC00466+miR-mimic). As revealed by CCK 8 assay, overexpressing miR-137 resulted in a significant decrease in cell viability, while such effect was significantly reversed when miR-137 and LINC00466 were simultaneously overexpressed (Figure 4A). In terms of cell migration and invasion abilities, they were considerably decreased after overexpression of miR-137, while their abilities were revised when miR-137 and LINC00466 were simultaneously overexpressed (Figure $4 \mathrm{~B}$ and C). Finally, we tested the effects of miR-137 on LINC00466attenuated glioma cell sensitivity to TMZ treatment. As shown in Figure 4D, down-regulation of miR-137 expression significantly influenced LINC00466-mediated glioma cell sensitivity to TMZ treatment (Figure 4D). Collectively, these findings illustrated that LINC00466 might regulated glioma cell proliferation, migration, invasion and sensitivity to TMZ by binding to miR-137. 


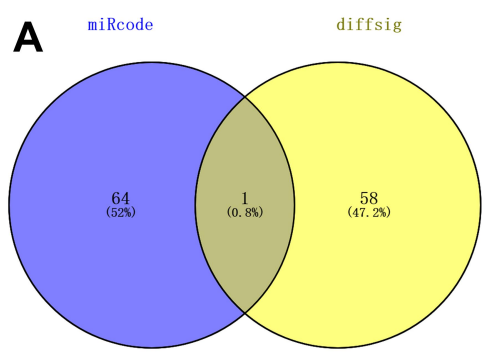

B

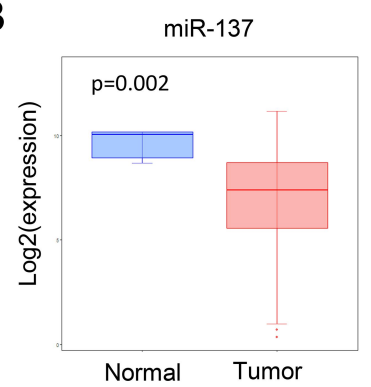

C

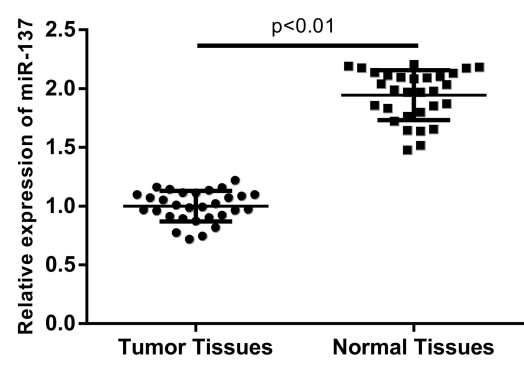

D

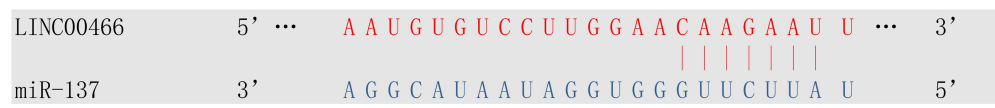

$\mathbf{E}$

$\mathbf{F}$
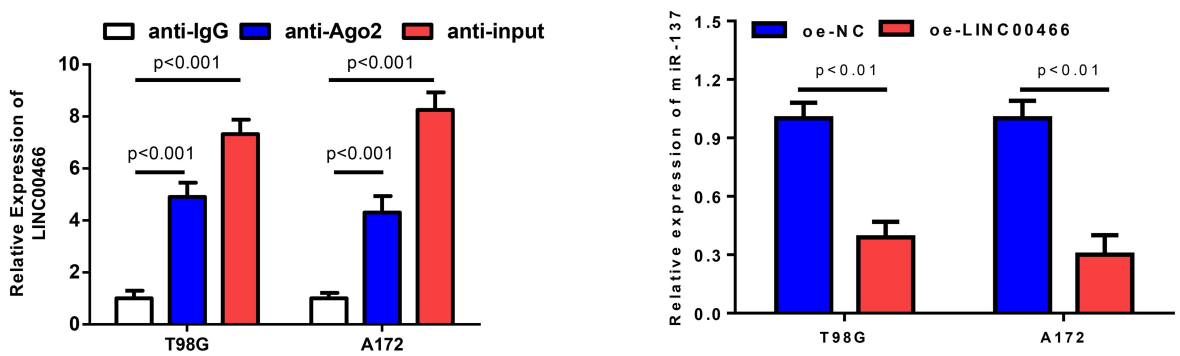

Figure 3 MiR-137 is the direct target of LINC00466. (A) VENNY plot of the predicted target miRNAs of LINC00466; (B) Differential expression of miR-137 in Tumor and Normal of TCGA-GBM/LGG; (C) The expression level of miR-137 in tissue of glioma and adjacent normal tissues; (D) The target binding site between miR-137 and LINC00466 and the results of dual luciferase assay; (E) The co-immunoprecipitation of LINC00466 and miR-137 in T98G and AI72 cell lines was evaluated by RIP assay; (F) The relative expression of miR-137 in T98G and AI72 cell lines after overexpressing LINC00466.

\section{LINC00466 Promotes PPPIRI4B}

\section{Expression by Inhibiting miR-I 37}

To find the mRNAs that were involved in LINC00466/miR137 regulation of glioma activity, integrated bioinformatical analysis (miRTarBase, TargetScan and mirDIP) were used to find genes. We identified 2 potential miR-137 target mRNAs, PPP1R14B and EZH2 (Figure 5A). Of which, PPP1R14B were high expression in tumor tissue (Figure $5 \mathrm{~B}$ and $\mathrm{D}$ ). The survival analysis showed that the survival time of patients with high expression of PPP1R14B was significantly lower than that of patients with low expression of PPP1R14B (Figure 5C). TargetScan database was employed and found that there existed potential binding site of miR-137 on PPP1R14B 3'UTR. Dual-luciferase reporter gene assay illustrated that upregulation of miR-137 could inhibit the luciferase activity of PPP1R14B-WT but had no impact on PPP1R14B-MUT, suggesting miR-137 could target PPP1R14B (Figure 5E). RIP assay confirmed the interaction between PPP1R14B and miR-137 in T98G cells (Figure 5F). In addition, we detected the effect of LINC00466 and miR-137 on the expression level of PPP1R14B in T98G cell lines, showing that PPP1R14B was significantly increased in cells upon overexpression of LINC00466. The PPP1R14B was significantly decreased in cells upon overexpression of miR-137 (Figure 5G). These results showed that LINC00466 was very likely to competitively bind to miR-137 with PPP1R14B to regulate occurrence and development of glioma.

Furthermore, we also explored LINC00466/miR-137/ PPP1R14B axis on the biological function of glioma cells. CCK8 and transwell assays revealed that silencing PPP1R14B and overexpression of miR-137 could suppress the promotion of proliferation and metastasis of glioma cells 
A

T98G

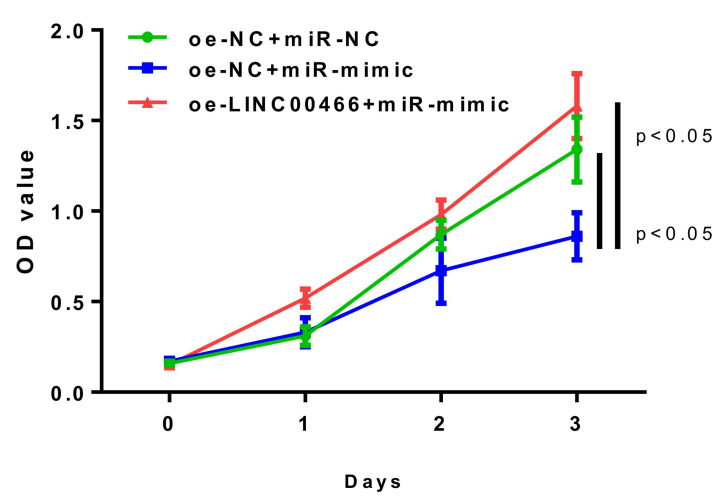

B

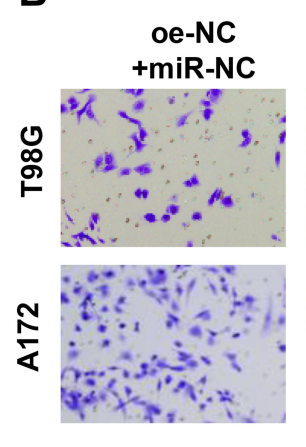
C oe-NC +miR-NC

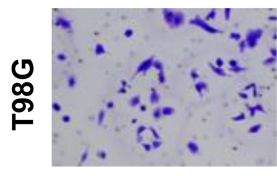

$\frac{\text { N }}{4}$

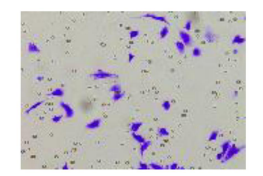

D

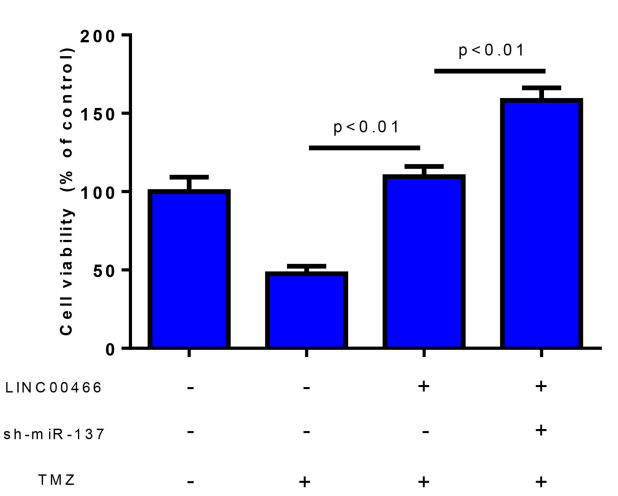

A172

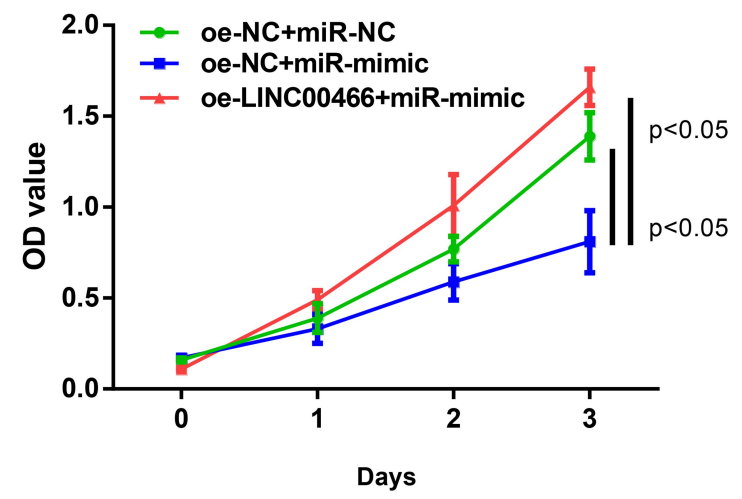

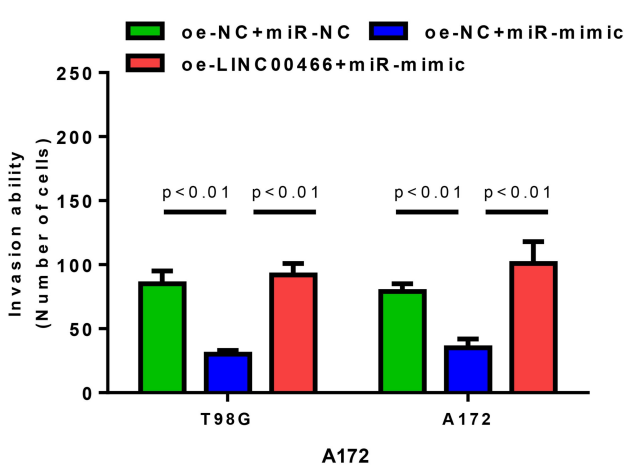

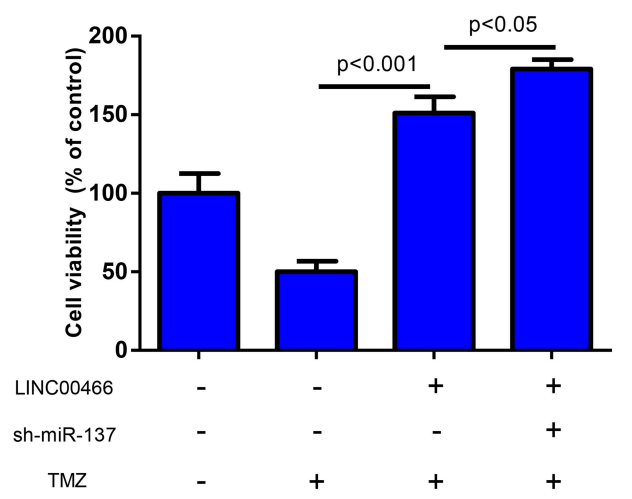

Figure 4 LINC00466 regulates glioma cell activity and sensitivity to TMZ treatment by targeting miR-137. (A) The viability, (B and C) migration combined with invasion abilities of T98G and AI72 cells in different treatment groups were measured by CCK8 assay and Transwell assay; (D) Effects of LINC00466 and miR-137 on resistance of glioma cells to TMZ (200uM). 
A

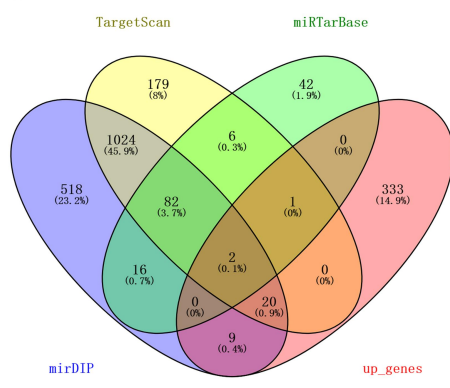

D

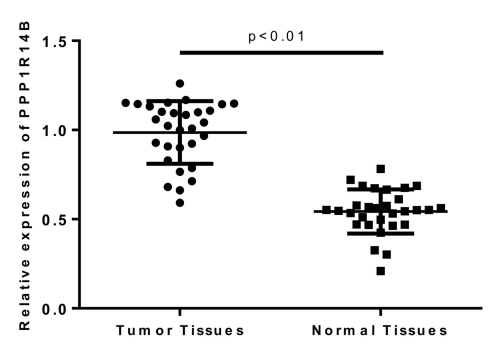

F

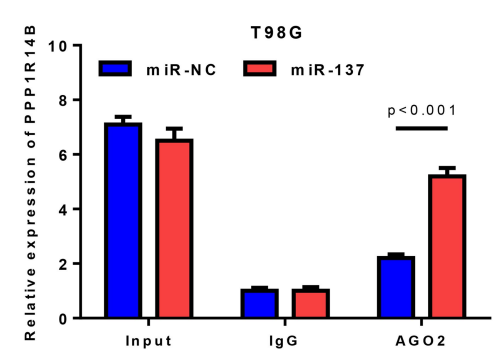

B

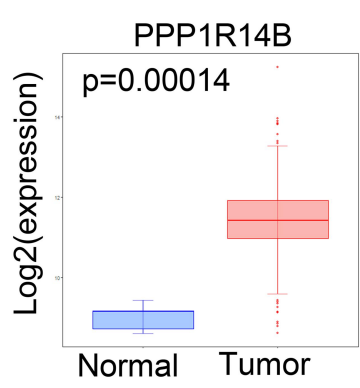

E

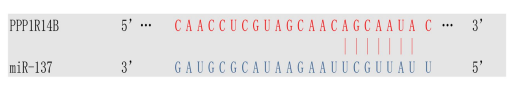

G

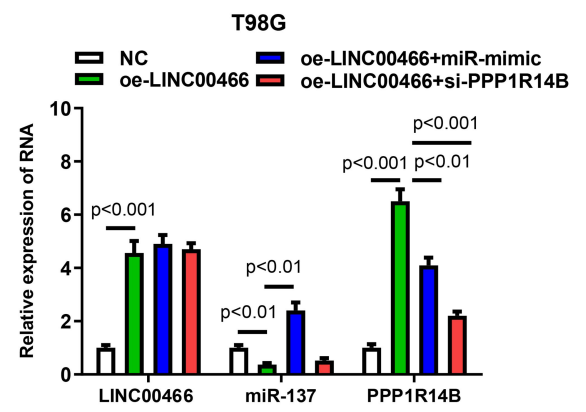

Figure 5 LINC00466 facilitates PPPIRI4B expression by inhibiting miR-I37. (A) VENNY plot of the predicted target mRNAs of miR-I37; (B) Differential expression of miR-137 in Tumor and Normal of TCGA-GBM/LGG; (C) The survival curves of PPPIRI4B expression level to the prognosis of patients, high-expression group was defined as red line, and low-expression group was defined as blue line; (D) The expression level of PPPIRI4B in glioma tissues and adjacent normal tissues; (E) The target binding site between miR-I37 and PPPIRI4B and the results of dual luciferase assay; (F) The co-immunoprecipitation of miR-I37 and PPPIR I4B in T98G cells was evaluated by RIP assay; (G) The relative expression levels of LINC00466, miR-I37 and PPPIRI4B in different groups of T98G cells.

by up-regulation of LINC00466 (Figure 6A, C and D). The impaction of LINC00466/miR-137/PPP1R14B axis on the sensitivity of glioma cell to TMZ showed LINC00466 and PPP1R14B could promote the resistance of glioma cell to TMZ. The results of miR-137 were opposite (Figure 6B). Taken together, these findings suggested LINC00466 might promoted the expression of PPP1R14B to regulate cell activity and resistance to TMZ treatment of glioma cells by competitively binding to miR-137.

\section{In vivo Experiments Prove That Overexpressing LINC00466 Promotes Glioma Tumor Growth}

To investigate the role of LINC00466 in glioma tumor growth in vivo, T98G cell line with lentivirus-coated
LINC00466 overexpression plasmid was inoculated into nude mice (oe-LINC00466 group), and the cell line with blank lentivirus plasmid was used as oe-NC (oe-NC group). The results displayed that LINC00466 overexpression noticeably fostered tumor growth (Figure 7A). Besides, the tumor size was significantly larger in oe-LINC00466 group compared with oe-NC group (Figure 7B). These findings noted that LINC00466 had a promoting effect on tumor growth of glioma. In addition, we also detected the expression levels of LINC00466/miR-137/PPP1R14B in the tumors. As shown in Figure 7C, we discovered that compared to the oe-NC, LINC00466 and PPP1R14B expression levels were markedly enhanced and miR-137 expression was decreased. In the treatment group of oe-LINC00466, the protein expression levels of PPP1R14B, N-cadherin, Vimentin, $\beta$-catenin, MMP-2, MMP9 and Cyclind1 were 
A

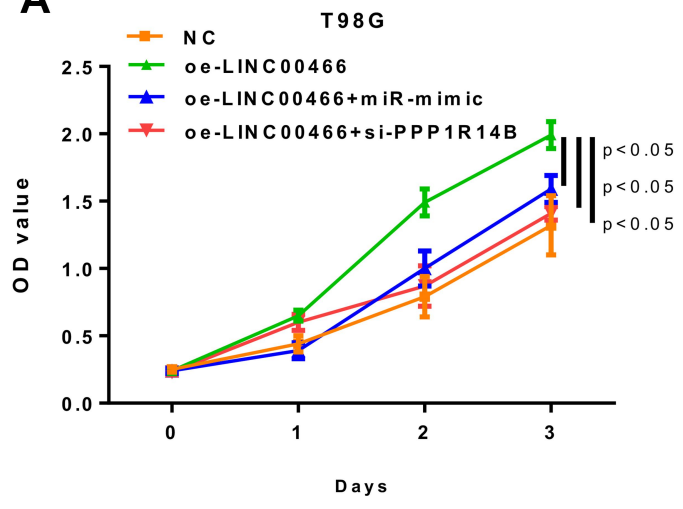

C

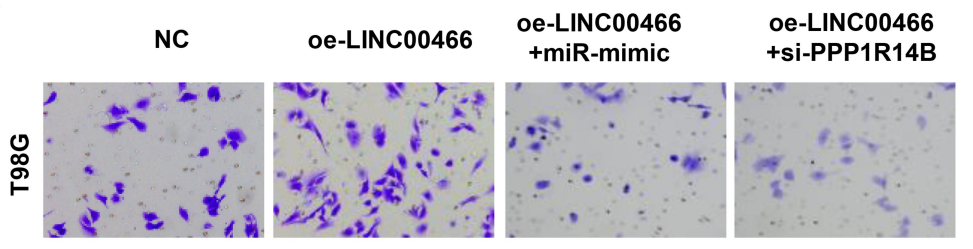

D

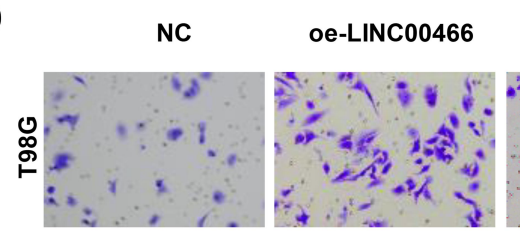

B
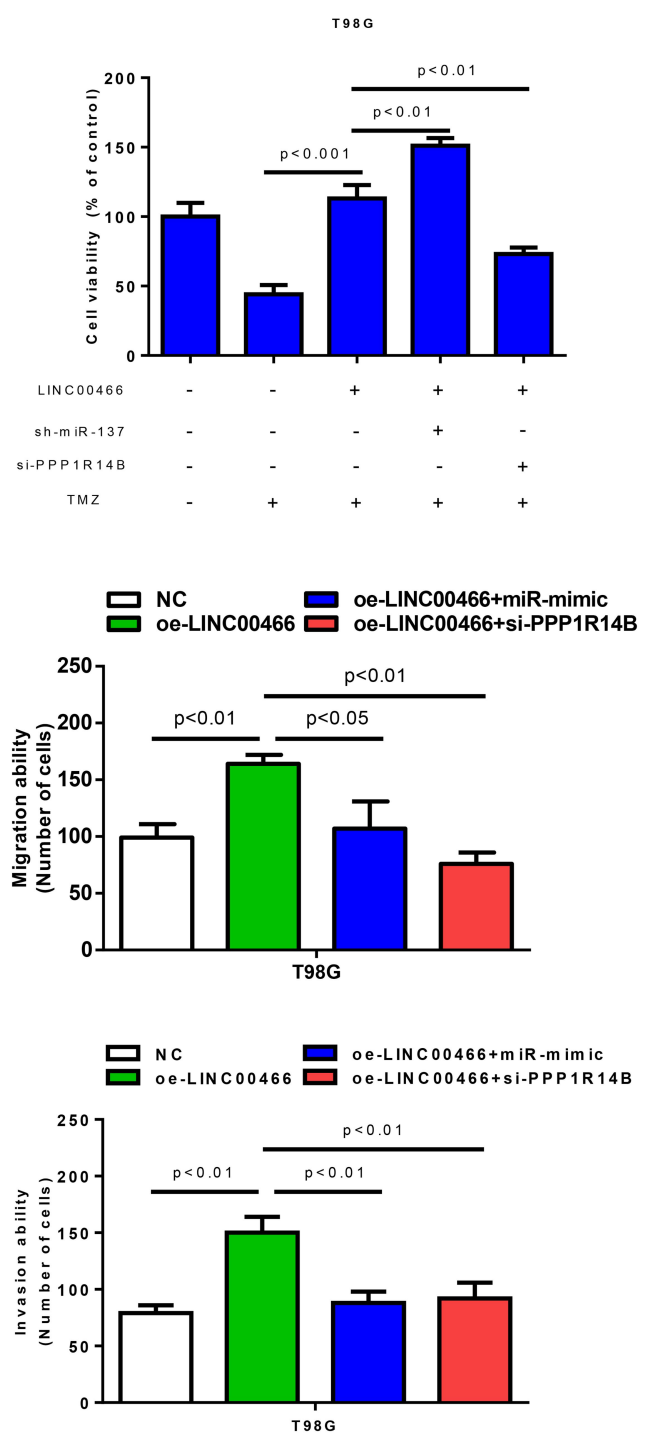

Figure 6 The effects on glioma cell activity and sensitivity to TMZ treatment by LINC00466/miR-I37/PPPIRI4B axis. (A) The viability abilities of T98G cells in different treatment groups were measured by CCK8 assay; (B) Effects of LINC00466/miR-I37/PPPIRI4B axis on cell resistance to TMZ (200uM); (C and D) The cell migration and invasion abilities of T98G cells in different treatment groups.

higher than the expression levels of these proteins in oe-NC (Figure 7D). The expression level of E-cadherin was opposite. IHC was performed to measure the expression of PPP1R14B and proliferation-related protein $\mathrm{Ki} 67$ in nude mice tissues of oe-NC and oe-LINC00466 groups, and it was found that the expression levels of PPP1R14B and $\mathrm{Ki}-$ 67 were evidently increased upon overexpression of LINC00460 (Figure 7E). Collectively, these findings demonstrated that overexpression of LINC00466 could promote tumor growth of glioma.

\section{Discussion}

LncRNAs are RNAs which include more than 200 nucleotides and lncRNAs lack open reading frames. ${ }^{20}$ In recent years lncRNAs have been reported participated in the development of tumors ${ }^{21}$ and other diseases. ${ }^{22}$ The pathogenesis of glioma is complex, the prognosis is poor, the mortality is high, and the effect of operation, radiotherapy and chemotherapy is poor for glioma. Although the pathogenesis of glioma is still unclear, more and more evidence shows that many lncRNAs and miRNAs are involved in the pathogenesis of glioma. ${ }^{23}$ For our study, we proved that LINC00466 was highly expressed. LINC00466 could improve the activity of glioma cells. In addition, overexpression of LINC00466 could contribute to knockdown of miR-137 and regulate downstream gene PPP1R14B expression through sponging miR-137. We also proved that miR-137 could inhibit the progression of tumor by 

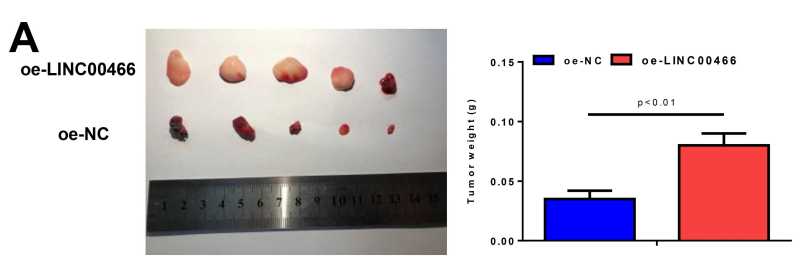

B

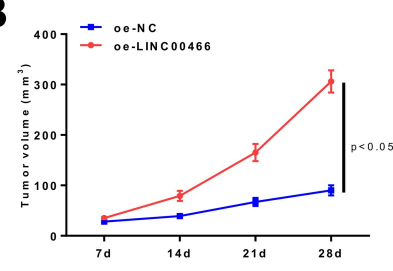

D

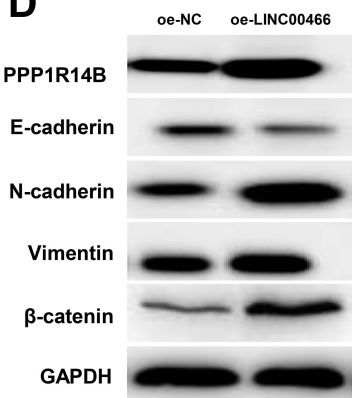

E

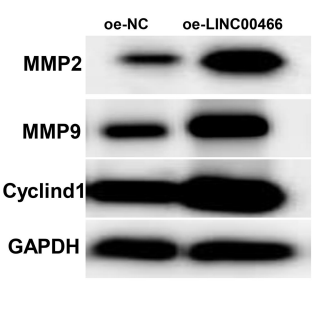

oe-NC

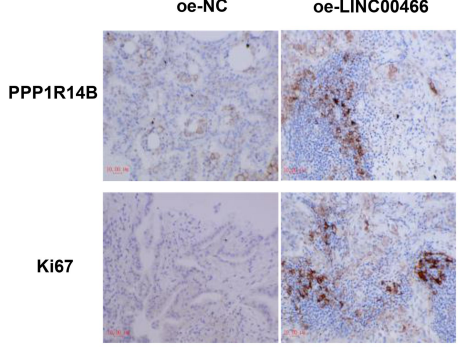

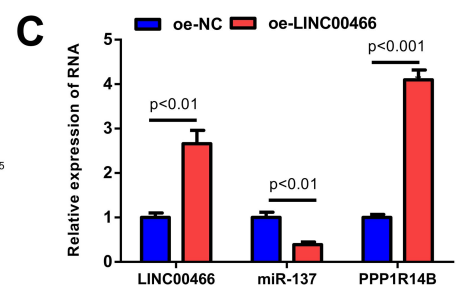

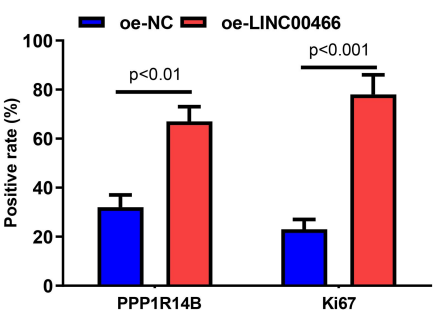

Figure 7 Overexpressing LINC00466 promotes tumor growth of glioma in nude mice. (A) Tumor weight and (B) tumor size in oe-NC and oe-LINC00466 groups; (C) The expression levels of LINC00466, mir-I37 and PPPIRI4B in the tumors; (D) The protein levels of PPPIRI4B, proteins which were related to EMT and proteins which were related to proliferation in tumor tissues were measured by WB assay (E) The expression levels of PPPIRI4B and Ki67 in tumor tissues were detected by IHC assay (IOum).

decreasing the expression of PPP1R14B. The results of nude mice study also proved that the tumor growth could be promoted when the expression of LINC00466 was elevated. Thus, the LINC00466/miR-137/PPP1R14B axis could increase the activity of glioma cells and sensitivity of glioma cell to TMZ, which implied these genes could be regarded as biomarkers and therapeutic target in the treatments of glioma.

In recent years, LINC00466 has been found to be highly expressed in lung squamous cell carcinoma, ${ }^{24}$ breast cancer ${ }^{19,25}$ and lung adenocarcinoma. ${ }^{18}$ In lung adenocarcinoma, Ma et al reported that the expression of LINC00466 in cancer tissues and cell lines was significantly higher than that in the corresponding adjacent normal tissues and normal bronchial epithelial cell lines. ${ }^{18}$ However, the expressive status of LINC00466 in glioma is lack. The experiments of our study first showed that the expression of LINC00466 in glioma tissues and cell lines was significantly increased compared with adjacent normal tissue pairs and normal human brain astrocyte cell lines, which was consistent with the status of LINC00466 in other cancers. For clinical significance of LINC00466, it was significantly associated with clinical stage in lung cancer. ${ }^{18}$ The relationship between LINC00466 expression and clinicopathological features was analyzed. We found LINC00466 expression level was significantly increased in WHO grade (III-IV) compared with WHO grade (I-II) of glioma samples. These studies consistently suggest that overexpression of LINC00466 may serve as a biomarker for the diagnosis of glioma patients.

In the process of gene transcription and translation, lncRNAs and miRNAs play important roles. The interaction between lncRNA and miRNA is called competitive endogenous RNA (ceRNAs). ${ }^{26,27}$ In glioma, some lncRNAs play the function of an endogenous "sponge" for miRNAs to influence malignant progression of glioma. Li and his partners found NEAT1 could regulate miR-152-3p/CCT6A axis to promote the progression of glioma. ${ }^{28}$ LINC00294 has also been found negatively modulated the activity of glioma cells via influence the expression of miR-1278. ${ }^{29}$ In this work, LINC00466 overexpression influenced the proliferation, migration, invasion of glioma and cell resistance to TMZ treatment. Another fundamental finding was that LINC00466 had the ability to bind to miR-137. Some researchers have reported miR-137 might inhibited tumorigenesis and this miRNA could be regulated by IncRNA-XIST and NCK1AS1. ${ }^{13,30}$ The results of our study also showed the expression of miR-137 was obviously decreased in tissues of glioma compared with the tissues of normal non-tumor. Upregulation of LINC00466 inhibited miR-137 expression and regulated the biological function that played by miR137. These results showed miR-137 as a negative regulator in glioma and it could also be regulated by LINC00466.

PPP1R14B is required for cell migration and retraction, which is an inhibitor of protein-phosphatase $1 .^{31}$ The abnormal expression of PPP1R14B has been proved in 
some types of cancer including melanoma and ovarian clear cell carcinoma. ${ }^{31,32}$ PPP1R14B was also thought to have relationship with the development of tumor. In this study, RIP combined with the experiment of luciferase reporter assay verified PPP1R14B was direct target of miR-137. Furthermore, the results of our research proved the up-regulation of LINC00466 promoted the expression of PPP1R14B by downregulating miR-137. These results implied that LINC00466 promoted the malignant progression and resistance to $\mathrm{TMZ}$ of glioma by regulating miR137/PPP1R14B axis.

\section{Conclusion}

In conclusion, our study predicted that the LINC00466/ miR-137/PPP1R14B signaling axis was closely linked to the malignant progression and resistance to TMZ of glioma, and we also confirmed that LINC00466 played a positive regulatory role in glioma cells. MiR-137 was the target of LINC00466 and PPP1R14B was the target of miR-137. Overexpression of LINC00466 increased the expression of PPP1R14B, promoted the proliferation, metastasis and cell resistance to TMZ of glioma. These findings lay a foundation for the exploration on the novel targeted therapy of glioma.

\section{Highlights}

1. LINC00466 and PPP1R14B were up-regulated in glioma tissues and cells, miR-137 was downregulated in glioma tissues and cells.

2. Overexpression of LINC00466 promoted glioma cells progression and resistance to TMZ.

3. LINC00466 promoted glioma progression by regulating miR-137.

4. LINC00466 targeted miR-137 to elevate the expression of PPP1R14B.

5. LINC00466 facilitated tumor growth in vivo.

\section{Abbreviations}

IncRNA, long non-coding RNA; miR-137, microRNA-137; PPP1R14B, protein phosphatase 1 regulatory subunit 14B; qRT-PCR, quantitative real-time PCR; RIP, RNA binding protein Immunoprecipitation; CCK8, cell counting kit-8; TMZ, temozolomide; ceRNA, competitive endogenous RNA; MRE, miRNA responsible elements; DMEM, Dulbecco's modified Eagle medium; WB, Western Blot; RIPA, radio immune precipitation assay; PVDF, polyvinylidene fluoride; BSA/TBST, bovine serum albumin or TrisBuffered Saline Tween; HRP, Horseradish peroxidase; ECL, electrochemiluminescence; FISH, fluorescence in situ hybridization; IHC, immunohistochemical; SD, standard deviation; ceRNAs, competitive endogenous RNA; GBM, glioblastoma multiforme; LGG, low grade glioma.

\section{Ethics Approval and Consent to Participate}

This study was approved by the Ethics Committee of the second affiliated hospital of Zhejiang university school of medicine (Approval No.2020549), and written informed consent was obtained from all patients. The ethics committee of the Second Affiliated Hospital of Zhejiang University have consented the animal research (Approval No. 2020106).

\section{Acknowledgments}

We acknowledge all the patients who have participated in the research.

\section{Funding}

This work was approved by National Natural Science Foundation of China (NO.81702462).

\section{Disclosure}

The authors report no conflicts of interest in this work.

\section{References}

1. Flavahan WA, Drier Y, Liau BB, et al. Insulator dysfunction and oncogene activation in IDH mutant gliomas. Nature. 2016;529 (7584):110-114. doi: $10.1038 /$ nature 16490

2. Turcan S, Rohle D, Goenka A, et al. IDH1 mutation is sufficient to establish the glioma hypermethylator phenotype. Nature. 2012;483 (7390):479-483. doi:10.1038/nature10866

3. Cuddapah VA, Robel S, Watkins S, Sontheimer H. A neurocentric perspective on glioma invasion. Nat Rev Neurosci. 2014;15 (7):455-465. doi:10.1038/nrn3765

4. Belliveau JG, Bauman G, Macdonald DR. Detecting tumor progression in glioma: current standards and new techniques. Expert Rev Anticancer Ther. 2016;16(11):1177-1188. doi:10.1080/14737140.20 16.1240621

5. Han Y, Wu Z, Wu T, et al. Tumor-suppressive function of long noncoding RNA MALAT1 in glioma cells by downregulation of MMP2 and inactivation of ERK/MAPK signaling. Cell Death Dis. 2016;7:e2123. doi:10.1038/cddis.2015.407

6. Rynkeviciene R, Simiene J, Strainiene E, et al. Non-Coding RNAs in Glioma. Cancers (Basel). 2018;11(1):17. doi:10.3390/cancers11010 017

7. Li Z, Xie X, Fan X, Li X. Long non-coding RNA MINCR regulates $\mathrm{miR}-876-5 \mathrm{p} / \mathrm{GSPT} 1$ axis to aggravate glioma progression. Neurochem Res. 2020;45(7):1690-1699. doi:10.1007/s11064-020-03029-8

8. Zheng YY, Xie J, Xu XM et al. LncRNA DDX11-AS1 Exerts Oncogenic Roles in Glioma Through Regulating miR-499b-5p/ RWDD4 Axis. Onco Targets Ther. 2021;14:157-164. doi:10.2147/ OTT.S278986 
9. Huang L, Li X, Ye H, et al. Long non-coding RNA NCK1-AS1 promotes the tumorigenesis of glioma through sponging microRNA-138-2-3p and activating the TRIM24/Wnt/beta-catenin axis. J Exp Clin Cancer Res. 2020;39(1):63. doi:10.1186/s13046020-01567-1

10. Hu T, Wang F, Han G. LncRNA PSMB8-AS1 acts as ceRNA of miR-22-3p to regulate DDIT4 expression in glioblastoma. Neurosci Lett. 2020;728:134896. doi:10.1016/j.neulet.2020.134896

11. Meng X, Deng Y, Lv Z, et al. LncRNA SNHG5 promotes proliferation of glioma by regulating miR-205-5p/ZEB2 axis. Onco Targets Ther. 2019;12:11487-11496. doi:10.2147/OTT.S228439

12. Liu ZZ, Tian YF, Wu H, Ouyang SY, Kuang WL. LncRNA H19 promotes glioma angiogenesis through miR-138/HIF-1alpha/VEGF axis. Neoplasma. 2020;67(1):111-118. doi:10.4149/neo_2019_19 $0121 \mathrm{~N} 61$

13. Chen M, Cheng Y, Yuan Z, Wang F, Yang L, Zhao H. NCK1-AS1 increases drug resistance of glioma cells to temozolomide by modulating miR-137/TRIM24. Cancer Biother Radiopharm. 2020;35 (2):101-108. doi:10.1089/cbr.2019.3054

14. Chen KC, Chen PH, Ho KH, et al. IGF-1-enhanced miR-513a-5p signaling desensitizes glioma cells to temozolomide by targeting the NEDD4L-inhibited Wnt/beta-catenin pathway. PLoS One. 2019;14 (12):e0225913. doi:10.1371/journal.pone.0225913

15. Li Z, Zhang J, Zheng H, et al. Modulating lncRNA SNHG15/CDK6/ miR-627 circuit by palbociclib, overcomes temozolomide resistance and reduces M2-polarization of glioma associated microglia in glioblastoma multiforme. J Exp Clin Cancer Res. 2019;38(1):380. doi:10.1186/s13046-019-1371-0

16. Voce DJ, Bernal GM, Wu L, et al. Temozolomide treatment induces lncRNA MALAT1 in an NF-kappaB and p53 codependent manner in glioblastoma. Cancer Res. 2019;79(10):2536-2548. doi:10.1158/ 0008-5472.CAN-18-2170

17. Ho WL, Che MI, Chou $\mathrm{CH}$, et al. B3GNT3 expression suppresses cell migration and invasion and predicts favorable outcomes in neuroblastoma. Cancer Sci. 2013;104(12):1600-1608. doi:10.1111/ cas. 12294

18. Ma T, Hu Y, Guo Y, Yan B. Tumor-promoting activity of long noncoding RNA LINC00466 in lung adenocarcinoma via miR-144regulated HOXA10 axis. Am J Pathol. 2019;189(11):2154-2170. doi:10.1016/j.ajpath.2019.06.014

19. Wang JJ, Huang YQ, Song W, et al. Comprehensive analysis of the IncRNA-associated competing endogenous RNA network in breast cancer. Oncol Rep. 2019;42(6):2572-2582. doi:10.3892/or.2019.7374

20. Ponting CP, Oliver PL, Reik W. Evolution and functions of long noncoding RNAs. Cell. 2009;136(4):629-641. doi:10.1016/j. cell.2009.02.006
21. Sun WH, Jiang CQ, Ji Y, et al. Long Noncoding RNAs: New Regulators of Resistance to Systemic Therapies for Gastric Cancer. Biomed Res Int. 2021;2021:8853269. doi:10.1155/2021/ 8853269

22. De Vincentis A, Rahmani Z, Muley M, et al. Long noncoding RNAs in nonalcoholic fatty liver disease and liver fibrosis: state-of-the-art and perspectives in diagnosis and treatment. Drug Discov Today. 2020;25(7):1277-1286. doi:10.1016/j.drudis.2020.05.009

23. Zheng SQ, Qi Y, Wu J, et al. CircPCMTD1 acts as the sponge of miR-224-5p to promote glioma progression. Front Oncol. 2019;9:398. doi:10.3389/fonc.2019.00398

24. Yao Y, Zhang T, Qi L, et al. Competitive endogenous RNA network construction and comparison of lung squamous cell carcinoma in smokers and nonsmokers. Dis Markers. 2019;2019:5292787. doi:10.1155/2019/5292787

25. Gao C, Li H, Zhuang J, et al. The construction and analysis of ceRNA networks in invasive breast cancer: a study based on The Cancer Genome Atlas. Cancer Manag Res. 2019;11:1-11. doi:10.2147/CMAR.S182521

26. Xue M, Zhuo Y, Shan B. MicroRNAs, long noncoding RNAs, and their functions in human disease. Methods Mol Biol. 2017;1617:1-25.

27. Mao Y, Liu R, Zhou $H$, et al. Transcriptome analysis of miRNA-lncRNA-mRNA interactions in the malignant transformation process of gastric cancer initiation. Cancer Gene Ther. 2017;24 (6):267-275. doi:10.1038/cgt.2017.14

28. Li B, Lu X, Ma C, et al. Long non-coding RNA NEAT1 promotes human glioma tumor progression via miR-152-3p/CCT6A pathway. Neurosci Lett. 2020;732:135086. doi:10.1016/j.neulet.2020.135086

29. Zhou X, Lv L, Zhang Z, Wei S, Zheng T. LINC00294 negatively modulates cell proliferation in glioma through a neurofilament medium-mediated pathway via interacting with miR-1278. J Gene Med. 2020;22:e3235. doi:10.1002/jgm.3235

30. Sun Y, Lv B, Zhang X. Knock-down of LncRNA-XIST induced glioma cell death and inhibited tumorigenesis by regulating miR-137/SLC1A5 axis-mediated ROS production. Naunyn Schmiedebergs Arch Pharmacol. 2020. doi:10.1007/s00210-02001831-3

31. Worley MJ Jr, Liu S, Hua Y, et al. Molecular changes in endometriosis-associated ovarian clear cell carcinoma. Eur $J$ Cancer. 2015;51(13):1831-1842. doi:10.1016/j.ejca.2015.05.011

32. Miquel-Serra L, Hernandez D, Docampo MJ, Bassols A. Differential expression of endoglin in human melanoma cells expressing the V3 isoform of versican by microarray analysis. Mol Med Rep. 2010;3 (6):1035-1039. doi:10.3892/mmr.2010.357
OncoTargets and Therapy

\section{Publish your work in this journal}

OncoTargets and Therapy is an international, peer-reviewed, open access journal focusing on the pathological basis of all cancers, potential targets for therapy and treatment protocols employed to improve the management of cancer patients. The journal also focuses on the impact of management programs and new therapeutic

Submit your manuscript here: https://www.dovepress.com/oncotargets-and-therapy-journal agents and protocols on patient perspectives such as quality of life, adherence and satisfaction. The manuscript management system is completely online and includes a very quick and fair peer-review system, which is all easy to use. Visit http://www.dovepress.com/ testimonials.php to read real quotes from published authors. 